\title{
THE INFLUENCE OF METHOD OF POINT COLLECTION ON RESULTS WITH THE USE OF A MEASURING ARM
}

\author{
Eugeniusz Ratajczyk ${ }^{1)}$, Michał Rak $^{2)}$, Tomasz Kowaluk ${ }^{2)}$ \\ 1) University of Ecology and Management in Warsaw, Wawelska 14,02-061 Warsaw, Poland (هerat@mchtr.pw.edu.pl) \\ 2) Warsaw University of Technology, Faculty of Mechatronics, A. Boboli 8,02-525 Warsaw, Poland (M.Rak@mchtr.pw.edu.pl, \\ T.Kowaluk@mchtr.pw.edu.pl)
}

\begin{abstract}
Comparison studies of different measurement methods using a Coordinate Measuring Arm are presented. Studies were divided into two parts. The first was point measurements of contact and pseudo-scanning contact measurements. The second part consisted of point measurements of contact and non-contact scanning measurements. Contact research (point measurements and the pseudo-scanning) were accomplished with the use of PowerINSPECT software, whereas non-contact with use of Focus Handheld and Focus Inspection software. Handheld Focus was used to collect a point cloud and its processing, while the detection of set elements was made using the second software from the group of Focus. According to the developed procedure for both parts sample elements with known nominal values were measured (available CAD model of object of research). It became the basis for examining whether there are statistically significant differences between results of different methods in both parts. Statistical comparison of measurement methods was carried out using four tests: Comparison of Means, Comparison of Standard Deviations, Comparison of Medians and a KolmogorovSmirnov Test.
\end{abstract}

Keywords: scanning, pseudo-scanning, measuring arm.

(C) 2012 Polish Academy of Sciences. All rights reserved

\section{Introduction}

The coordinate measuring technique, implemented by using Coordinate Measuring Machines (CMM's), is currently the most advanced section of geometrical quantities metrology. It allows to measure complex parts for the needs of machinery industry, especially automotive and aerospace [1]. Due to computerization, in processing of results of measurements as well as control, it allows to make measurements of objects in the rhythm of their production, which enables a direct impact on the quality of the manufacturing process and thereby on the quality of products. Coordinate Measuring Machines are in the assumption laboratory devices, although recently have appeared those of their constructional options which can be used in the manufacturing environment $[2,3]$. They are complemented by Coordinate Measuring Arms (portable CMM's) [4]. Measuring Arms, although characterized by a lower accuracy compared to Measuring Machines, have the advantage that they can be applied directly in the production and in terrain and can measure inside large objects.

Therefore they can be used in small- and medium-size manufacturing plants. In contrast to the Coordinate Measuring Machines, in Measuring Arms, during point collection angular coordinates from the rotary transducers (encoders) are read. Then coordinates of the point are transformed to the Cartesian system (x, y, z). Uncertainty depends on differences of coordinates of probing points used to calculate particular characteristic [5, 6]. In Measuring Arms accuracy is known. Knowledge about the accuracy includes usually catalog data related 
to the limit values of measurement errors. Therefore it was attempted to evaluate the accuracy of these two types of measurements on the example of a selected item. It was also examined whether there are statistically significant differences between point measurements and pseudo-scanning.

\section{Characteristics of measuring arm and laser scanner}

Studies were performed using a Metris Nikon Metrology Series MCA II [7] coordinate measuring arm which is in the equipment of the laboratory of the Institute of Metrology and Biomedical Engineering of Warsaw University of Technology (Fig. 1). This is a seven-axis portable measuring device designed to work in the environment of production, in laboratories. Because of applying a rechargeable battery and wireless communication it can also be used outdoors.

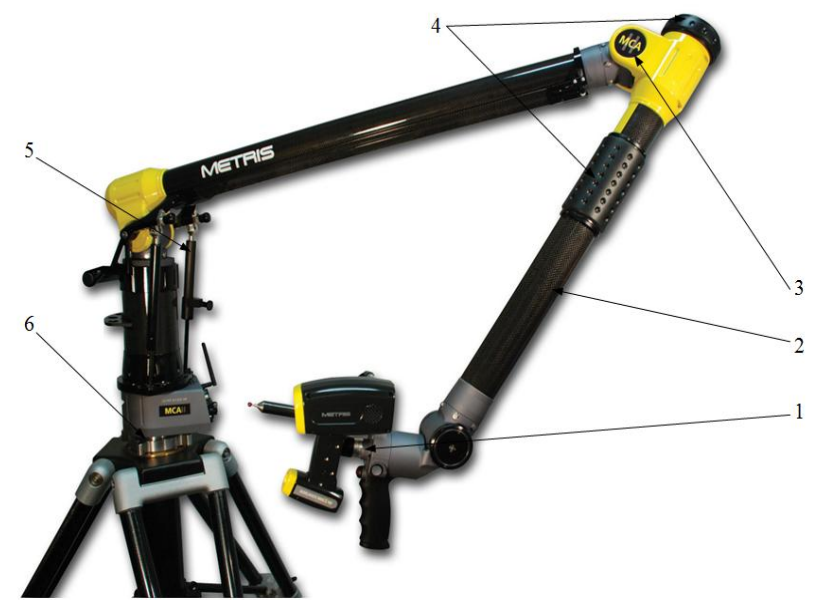

Fig. 1. Coordinate measuring arm.

The measuring head of measuring arm (Fig. 1) is equipped with two slots for probes and laser head installation, by applying a kinematic adapter. Installation is characterized by a high repeatability of $0.002 \mathrm{~mm}$. Each time after replacing the measuring probe, the arm recognizes the probe automatically. Additionally in the measuring head buttons for "collecting" or "deleting" data points are located. Tubes 2 in various arms are made of carbon fiber, which is characterized by high durability and resistance to temperature influences. Angle encoders 3 are installed in pairs at each joint. They enable the location of coordinates of the measuring probe. In the described arm there are seven pairs of angular encoders. Cartesian coordinates are calculated from the relation between the angular settings of the individual angular encoders and the distances between them. To improve comfort and increase ergonomics of the arm in the tube overlay Spin Grip on his shoulder and End Grip on his elbow 4 are mounted. Counterbalance Zero-G 5 consisting of two actuators is so constructed that it compensates the weight of the arm in the entire measuring range. Another characteristic element of the measuring arm is a mounting system 6 consisting of a threaded ring which allows mounting the arm to the tripod, magnetic or pneumatic bases.

The measuring arm has a measuring range of $2.4 \mathrm{~m}$. The accuracy of the arm expressed a permissible error specified by the manufacturer for the Single-Point Articulation Performance Test $0.028 \mathrm{~mm}$ and for the Volumetric Performance Test $0.040 \mathrm{~mm}$. These values are calculated on the basis of the American standard ASME B89.4.22-2004 [8].

For scanning measurements a METRIS - NIKON model MMC80 laser scanner was used. It works on the basis of triangulation, which means that the head generates a light strip, which 
after forming by the optical system is visible on the measured object. The image of this stripe is recreated on the photodetector by an optical system. In Fig. 2a a view of the scanner coupled with a contact probe is shown, and in Fig. $2 b$ there is an outline of the action. Main parameters of the scanner were shown in Table 1.
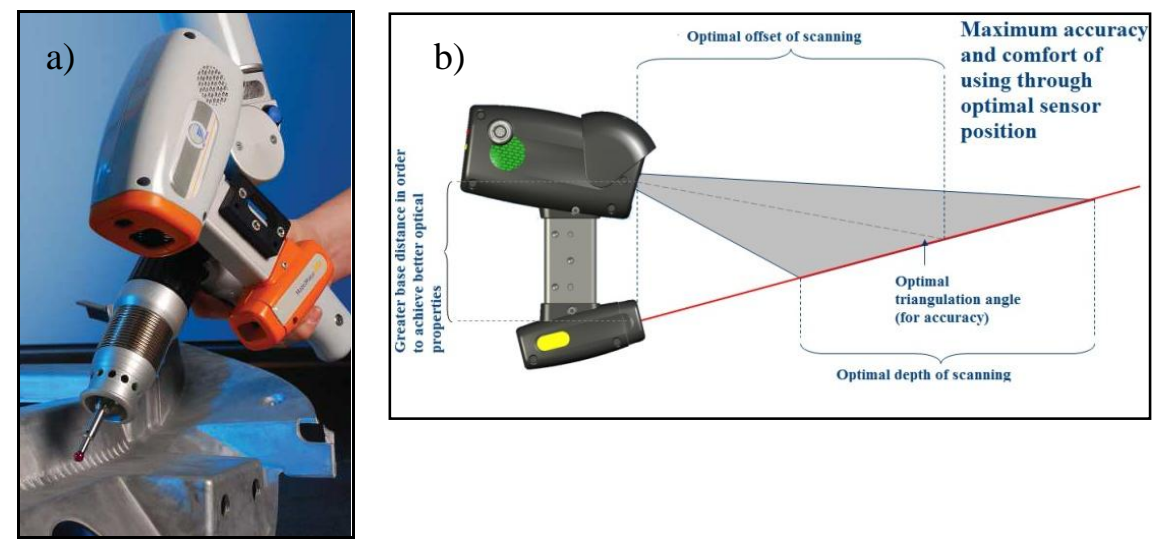

Fig. 2. Head of the Measuring Arm: a) view of the head with attached laser scanner, b) outline of action of the laser head.

Table 1. Parameters of the MMC80 scanner.

\begin{tabular}{|l|l|}
\hline Strip width $(\mathrm{Y})$ & $80 \mathrm{~mm}$ \\
\hline Start measuring range & $100 \mathrm{~mm}$ \\
\hline Measuring range $(\mathrm{Z})$ & $100 \mathrm{~mm}$ \\
\hline Accuracy $(1 \sigma)$ & $17 \mu \mathrm{m}$ \\
\hline Points per strip & 800 \\
\hline Maximum speed of scanning (strips per second) & 30 \\
\hline $\begin{array}{l}\text { Maximal number of the scanned points per } \\
\text { second }\end{array}$ & $24000 \mathrm{p} / \mathrm{s}$ \\
\hline Sensor weight $(\mathrm{g})$ & 395 \\
\hline
\end{tabular}

It appears from Table 1 that within one second the user can generate a maximum 30 lines about a width of $80 \mathrm{~mm}$, collecting 800 points from each line.

\section{Experiment}

In this paper two types of comparisons were presented. The first involves the comparison of point measurements of contact and the pseudo-scanning contact measurements. In this part the main goal was verification how the density of points affects the measurement. The second section compared the non-contact optical method - laser scanning and contact-point measurements. Non-contact measurements give a large number of points, but with low accuracy. On the other hand, the measurement is fast and does not affect the measured surface. It was taken to attempt a statistical comparison of results from both methods.

\section{Software}

For both parts of the comparison of three kinds of software was applied. For contact measurements - PowerINSPECT software, whereas to non-contact measurements a group of Focus software was applied. The Focus group includes: Focus Handheld and Focus Inspection. 


\subsection{PowerINSPECT}

PowerINSPECT is a measuring software which can cooperate with different types of devices working in coordinate technology [9]. It is used for an inspection of measured elements. In case of having a model CAD of the measured element it is possible to compare the compliance of measured points with corresponding points on the CAD model. In case of not-having the model CAD, it is possible to measure the geometry of the product without its quality assessment. The software enables also reporting, which is created on the fly. The report can have the form of a table, of graphical illustrations or statistical data. Results of measurements are also being shown in real time and measuring plans created during the measurement are memorized so that they can be used to control the next parts of the same forms and dimensions.

\subsection{Focus Handheld}

The Focus Handheld software enables scanning using the Coordinate Measuring Arm equipped with a laser scanner. In this software the cloud of points during scanning is collected, what is created in real time. In Focus Handheld unwanted pieces of collected cloud for example in case of scanning the fastening of the measured element can be removed. From the collected points a mesh of triangles is created. It is created on the basis of a greater number of points accumulated on the edges whereas on the surface without edges the density is lower. After creating the mesh of triangles, a program filling of not-scanned areas and a reduction in noise are possible.

\subsection{Focus Inspection}

The Focus Inspection software is used for geometrical analysis of the mesh of triangles created using the Focus Handheld software. With its use it is possible to detect demanded elements and to compare them with nominal values in case of having a CAD model of the measured object.

\section{Measuring procedure}

For comparative research of the accuracy of point measurements, contact pseudo-scanning and non-contact scanning, an element with simple geometry was chosen. It was a measuring cube made of Teflon with a mass of $1200 \mathrm{~g} \pm 5 \mathrm{~g}$ and dimensions $250 \mathrm{~mm} \times 110 \mathrm{~mm}$ x 45 $\mathrm{mm}$. Several features were measured, however for the purpose of this paper results of measurements of selected features are presented. Their nominal dimensions were marked at the view of the CAD model of the element and are presented in Fig. 3.

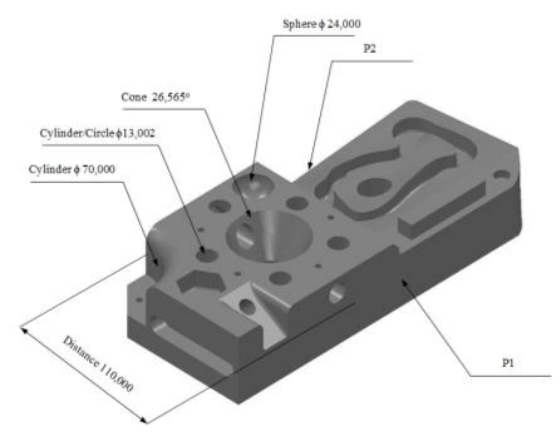

Fig. 3. Model of the measuring cube. 


\subsection{Comparison of point measurements and pseudo-scanning}

For the comparing analysis of two measurement methods, the following features were selected:

- circle with a nominal diameter of $13.002 \mathrm{~mm}$,

- sphere with a nominal diameter of $24.000 \mathrm{~mm}$,

- cone with a nominal angle of $26.565^{\circ}$,

- cylinder with a nominal diameter of $70.000 \mathrm{~mm}$.

In order to perform the measurement of established features in the PowerINSPECT software, action was performed according to instructions in the following order:

- measurement of circle, sphere, cone and cylinder,

- creating a report.

Nominal values are read from the CAD model. To allow statistical comparison of two measurement methods such as point measurement and pseudo-scanning, twenty-fold measurements of each of the selected elements for each method were made. During the collection of measurement points, rotations of encoders should be minimized (if smaller individual joints movements are made, better accuracy is obtained during the measurement).

Studies have shown that the highest repeatability and accuracy is obtained by setting the measuring probe in the same way as it is in coordinate measuring machines. In addition, the measurement should be performed in such a way that, whenever possible, not to stop contact of the stylus with the surface of the measured element.

For each geometric feature a constant number of measurement points was specified. For the point measurement of a circle 6 points were defined, while for the pseudo-scanning 30 points. For other geometric elements 15 and 75 points were adopted respectively. Poniatowska et al. [10] indicated that the result of a measurement depends on measurement parameters such as measuring tip's diameter and the number of measurement points - the sampling interval. Influence of the chosen measuring strategy was also confirmed by Bubnowicz et al. [11].

\subsection{Comparison of point measurements and scanning}

\subsubsection{Measuring procedure for contact measurements}

The aim of the measuring procedure for contact measurements was to present the actions required to measure the assumed features. Within this paper it was presented for:

- a cylinder with a nominal diameter of $13.002 \mathrm{~mm}$,

- a sphere with a nominal diameter of $24.000 \mathrm{~mm}$,

- the distance between planes P1 and P2 with a nominal value of $110.000 \mathrm{~mm}$.

In order to perform the measurement of established features in the PowerINSPECT software, action was performed according to instructions in the following order:

- measurement of planes, a cylindrical hole, sphere,

- appointment of dimension - dimension which is the distance between opposite planes is the resultant value - calculated on the basis of already measured elements,

- creating a report.

\subsubsection{Measuring procedure for non-contact measurements}

The scanning measurement is carried out using two kinds of Focus software: Focus Handheld and Focus Inspection. The procedure was therefore divided into two parts. 
Part of measurements carried out in the Focus Handheld software takes place according to the instructions below:

- qualification of the scanner,

- scan using Handheld software,

- removal of unwanted elements - at scanning the measured element a cloud of points was also collected from fastenings or the measuring table,

- creating a mesh of triangles - software with its creation leaves a larger concentration of points on a different kind of edges and a smaller one on the smooth surfaces,

- processing the mesh of triangles - the created mesh of triangles can contain tearing of a different kind, discontinuities or noise. It was necessary to remove them because they influenced negatively the result of measurement.

After processing the mesh of triangles one should save the file with extension *.sab2 in order to analyze it in the Focus Inspection software. In order to perform the measurement of established features in the Focus Inspection software they were acting according to the presented instruction:

- import of CAD model - there was the compared scanned element with it,

- defining measured features of the CAD model,

- import of created mesh of triangles - saved in Focus Handheld software,

- fitting the mesh of triangles to the CAD model,

- defining measured features on the mesh of triangles,

- comparison of the mesh of triangles with a nominal element,

- report creation - it contains features specified by the user.

\section{Results}

\subsection{Results of contact measurements and pseudo-scanning}

Based on the obtained results statistical parameters for each measured feature were calculated and are presented in Table 2 .

Table 2. Summary of measurement results.

\begin{tabular}{|l|l|l|}
\hline \multicolumn{3}{|c|}{ The results of measuring the diameter of a circle with a nominal value of $13.002 \mathrm{~mm}$} \\
\hline Parameter & Point measurements & Pseudo-scanning \\
\hline The mean value & 13.025 & 13.017 \\
\hline The standard deviation & 0.010 & 0.007 \\
\hline Range & 0.042 & 0.029 \\
\hline \multicolumn{2}{|c|}{ The results of measuring the diameter of a sphere with a nominal value of $24.000 \mathrm{~mm}$} \\
\hline Parameter & Point measurements & Pseudo-scanning \\
\hline The mean value & 24.038 & 24.020 \\
\hline The standard deviation & 0.020 & 0.010 \\
\hline Range & 0.072 & 0.037 \\
\hline \multicolumn{1}{|c|}{ The results of measuring the cone angle with a nominal value of 26.565} \\
\hline Parameter & Point measurements & Pseudo-scanning \\
\hline The mean value & 26.404 & 26.401 \\
\hline The standard deviation & 0.016 & 0.010 \\
\hline Range & 0.058 & 0.036 \\
\hline The results of measuring the diameter of a cylinder with a nominal value of $70.000 \mathrm{~mm}$ \\
\hline Parameter & Point measurements & Pseudo-scanning \\
\hline The mean value & 69.859 & 69.841 \\
\hline The standard deviation & 0.033 & 0.028 \\
\hline Range & 0.127 & 0.111 \\
\hline
\end{tabular}


Comparing the results of mean values for both measurement methods, it was found that in the case of three features: the circle, the sphere and the cone, lower values were obtained by measuring the pseudo-scanning. However, in the case of the cylinder measurement a smaller mean value was obtained from the point measurement. Differences between mean values ranged from 0.008 to $0.018 \mathrm{~mm}$ and they are not higher than the volumetric accuracy of the measuring arm of $\pm 0.040 \mathrm{~mm}$. The same relation exists when median values are compared. It is worth to mention that data collected in the measurement process include two separate components: the random and the deterministic. The smooth surface trend is represented by the deterministic errors which are spatially correlated, while the random errors are of a spatially random character $[12,13]$.

A comparative analysis of standard deviations shows that the measured pseudo-scanning is characterized by a smaller range of results. Differences between calculated values of standard deviation range from $0.003 \mathrm{~mm}$ for comparison of results of the measurement the circle, to $0.010 \mathrm{~mm}$ for comparison of results of the measurement of the sphere.

Comparison of the obtained range value showed that for each geometric feature the pseudo-scanning measurements are characterized by higher repeatability. Differences between calculated values of range are from $0.013 \mathrm{~mm}$ for the measurement of the circle to $0.035 \mathrm{~mm}$ when a sphere was measured.

Statistical tests available in the software are used for comparative analysis of a series of measuring samples coming from normal distribution. So the first step was to verify normal distribution of the results for each geometric feature. After importing the measurement results to Statgraphics software at first it was examined whether there are any differing points of statistical terms from the series using the chart "Box and Whisker Plot." Normality of the distribution is checked by the function "Tests for Normality", by comparing the obtained values of P-value for the four tests: Chi-square, Shapiro-Wilk, skewness and kurtosis. All test results showed the normality distribution of individual measurement series.

The next step was to perform comparative tests. Four comparative tests were selected: a comparison of mean values, standard deviations, medians and Kolomogorov-Smirnov test.

For two geometric features (circle and sphere), there were statistically significant differences at a confidence level of $95 \%$. For comparison, the results of the circle were negative in tests: comparing mean values, medians and Kolmogorov-Smirnov test. However, in the case of a comparison of measurement results of the sphere, all four tests showed significant differences between obtained results. Between obtained results of measurement of the angle of the cone and the diameter of the cylinder tests showed no statistical significant differences at a confidence level of $95 \%$.

Results of statistical tests were confirmed by the calculated mean values, standard deviations, range and medians. In the case of analysis of differences of mean values, results ranging from 0.008 to $0.018 \mathrm{~mm}$ were obtained. In the case of the standard deviations comparison, for each of selected features lower values for pseudo-scanning measurements were found. An identical relation was found when values of ranges were analyzed. Because during pseudo-scanning measurements the values of standard deviations and range are smaller than point measurements it was found that the pseudo-scanning measurements are characterized by higher repeatability.

\subsection{Point measurements and laser scanning}

\subsubsection{Results of contact measurements}

During researches a series of twenty-one measurements of established features was performed. Results of measurements were statistically analyzed in the STATGRAPHICS Plus 
software [14]. An important issue from the point of view of statistical analysis is to examine the normality of distribution. While checking whether the given sample comes from the normal distribution four tests were used: the Chi-square test, Shapiro-Wilk test, assessment of skewness and assessment of kurtosis. It appeared that one cannot reject the hypothesis that established features come from a normal distribution with $90 \%$ or higher level of confidence. For each feature the mean value together with the number of samples from which became marked, variance, standard deviation and range - difference between determined minimum and maximum were appointed. Results of these parameters for the studied features are shown in Table 3.

Table 3. Basic parameters of the contact studied features.

\begin{tabular}{|l|l|l|l|}
\hline & Cylinder, $\mathrm{mm}$ & Sphere, $\mathrm{mm}$ & Distance, $\mathrm{mm}$ \\
\hline nominal value & 13.002 & 24.000 & 110.000 \\
\hline number of samples & 21 & 19 & 19 \\
\hline mean from a series of 20 & 13.047 & 24.060 & 109.967 \\
\hline variance & 0.0001 & 0.0005 & 0.0005 \\
\hline standard deviation & 0.012 & 0.023 & 0.022 \\
\hline range & 0.049 & 0.079 & 0.078 \\
\hline
\end{tabular}

An important parameter in evaluating the uncertainty of measurement is the standard deviation $\sigma$. Its doubled value indicates that with a probability of $95 \%$ the real dimension is within the range $x_{\text {mean }} \pm 2 \sigma$ where $x_{\text {mean }}$ means the mean value of the dimension. Its value in the analyzed features is within the range $(0.024 \div 0.046) \mathrm{mm}$.

\subsubsection{Results of non-contact measurements}

In case of non-contact measurements a series of twenty-one measurements was also made and results were statistically analyzed.

After the confirmation that it is not possible to reject the hypothesis that the features being considered come from the normal distribution of $90 \%$ or higher level of confidence, a number of parameters describing the analyzed samples was appointed. They were presented in Table 4.

In non-contact measurements the value of the doubled standard deviation for examined features is within the range $(0.154 \div 0.200) \mathrm{mm}$.

Table 4. Basic parameters of the non-contact studied features.

\begin{tabular}{|l|l|l|l|}
\hline & Cylinder, $\mathrm{mm}$ & Sphere, $\mathrm{mm}$ & Distance, $\mathrm{mm}$ \\
\hline nominal value & 13.002 & 24.000 & 110 \\
\hline number of samples & 20 & 17 & 20 \\
\hline mean from a series of 20 & 12.869 & 23.927 & 109.913 \\
\hline variance & 0.0100 & 0.0060 & 0.0097 \\
\hline standard deviation & 0.100 & 0.077 & 0.099 \\
\hline range & 0.394 & 0.303 & 0.359 \\
\hline
\end{tabular}

\subsubsection{Comparison of the results of contact and non-contact research}

The aim of the research was to determine whether there are statistically significant differences between measurements performed on the Coordinate Measuring Arm in contact 
and non-contact scanning way. Using the statistical software STATGRAPHICS Plus, the same features were measured by two various methods and were being compared with themselves. To determine whether the differences were statistically significant, four tests were used: comparison of means, comparison of standard deviations, comparison of medians and $\mathrm{K}-\mathrm{S}$ test [14]. Below a table is presented (Table 5.) which determines the significance of differences for each feature according to mentioned tests.

Table 5. The results of measurements of both methods - level of significance.

\begin{tabular}{|l|l|l|l|}
\hline Name of test & $\begin{array}{l}\text { Cylinder } \\
\text { (contact/non-contact) }\end{array}$ & $\begin{array}{l}\text { Sphere } \\
\text { (contact/non-contact) }\end{array}$ & $\begin{array}{l}\text { Distance } \\
\text { (contact/non-contact) }\end{array}$ \\
\hline \multirow{2}{*}{$\begin{array}{l}\text { comparison of } \\
\text { means }\end{array}$} & $13.047 \mathrm{~mm} / 12.869 \mathrm{~mm}$ & $24.060 \mathrm{~mm} / 23.927 \mathrm{~mm}$ & $109.967 \mathrm{~mm} / 109.913 \mathrm{~mm}$ \\
& $\Delta \mathrm{D}=0.178 \mathrm{~mm}$ & $\Delta \mathrm{D}=0.133 \mathrm{~mm}$ & $\Delta \mathrm{L}=0.054 \mathrm{~mm}$ \\
& Significant difference & Significant difference & Significant difference \\
\hline comparison of & $0.012 \mathrm{~mm} / 0.100 \mathrm{~mm}$ & $0.023 \mathrm{~mm} / 0.077 \mathrm{~mm} /$ & $0.022 \mathrm{~mm} / 0.099 \mathrm{~mm} /$ \\
standard & $\Delta \sigma=0.088 \mathrm{~mm}$ & $\Delta \sigma=0.054 \mathrm{~mm}$ & $\Delta \sigma=0.077 \mathrm{~mm}$ \\
deviations & Significant difference & Significant difference & Significant difference \\
\hline \multirow{2}{*}{$\begin{array}{l}\text { comparison of } \\
\text { medians }\end{array}$} & $13.049 \mathrm{~mm} / 12.843 \mathrm{~mm}$ & $24.060 \mathrm{~mm} / 23.917 \mathrm{~mm}$ & $109.969 \mathrm{~mm} / 109.956 \mathrm{~mm}$ \\
& $\Delta \mathrm{M}=0.206 \mathrm{~mm}$ & $\Delta \mathrm{M}=0.143 \mathrm{~mm}$ & $\Delta$ M=0.013 mm \\
\hline \multirow{2}{*}{ K-S test } & Significant difference & Significant difference & Significant difference \\
& P-value $\approx 1.241 \mathrm{E}-7$ & P-value $\approx 8.628 \mathrm{E}-6$ & P-value $\approx 5.50706 \mathrm{E}-3$ \\
& Significant difference & Significant difference & Significant difference \\
\hline
\end{tabular}

As shown in Table 5 for any of the presented features the hypothesis that there were statistically significant differences between the results coming from contact and non-contact measurements performed on the Coordinate Measuring Arm cannot be rejected with 95\% confidence level.

\subsection{Uncertainty of measurements}

An integral part of the measurements is the determination of their uncertainty. Factors such as influence of the operator, environmental conditions and accuracy of the measuring device have an influence on the result of measurement. With regard to the mentioned factors the measuring equation is:

$$
D=I+P_{I P}+P_{I S}+P_{T}
$$

where: $\mathrm{D}$ - measured dimension, $\mathrm{I}$ - indication, $\mathrm{P}_{\mathrm{IP}}$ - correction of indication in contact measurements, $\mathrm{P}_{\mathrm{IS}}$ - correction of indication in non-contact measurements, $\mathrm{P}_{\mathrm{T}}$ - temperature correction. Uncertainty of temperature correction is expressed by equation [15]:

$$
\begin{gathered}
u_{\text {tempN }}=D * \sqrt{\begin{array}{l}
\left.u_{T M r a n}^{2}+u_{T M c a l}^{2}\right) \alpha_{M}^{2}+\left(u_{T N r a n}^{2}+u_{T N c a l}^{2}\right) \alpha_{N}^{2}+ \\
+\left(u_{\alpha M}\left(T_{M}-20^{\circ} C\right)\right)^{2}+\left(u_{\alpha N}\left(T_{N}-20^{\circ} C\right)\right)^{2}
\end{array}} \\
u\left(P_{T}\right)=\sqrt{u_{\text {tempN }}^{2}+u_{\text {temp } W}^{2}},
\end{gathered}
$$

where: $\mathrm{T}_{\mathrm{W}}, \mathrm{T}_{\mathrm{M}}, \mathrm{T}_{\mathrm{N}}-$ mean value of temperature (object, machine, gauge); $\mathrm{u}_{\alpha \mathrm{W}}, \mathrm{u}_{\alpha \mathrm{M}}, \mathrm{u}_{\alpha \mathrm{N}}-$ standard uncertainty of linear coefficient of thermal expansion(object, machine, gauge); $\alpha \mathrm{W}$, $\alpha \mathrm{M}, \alpha \mathrm{N}$ - coefficient of linear thermal expansion (object, machine, gauge); $\mathrm{u}_{\mathrm{TWtran}}, \mathrm{u}_{\mathrm{TM} t r a n}$, 
$\mathrm{u}_{\mathrm{TNtran}}$ - standard uncertainty of thermal conductivity for the thermometers used (object, machine, gauge); $\mathrm{u}_{\mathrm{TWcal}}, \mathrm{u}_{\mathrm{TMcal}}, \mathrm{u}_{\mathrm{TNcal}}$ - standard uncertainty of the indications of used thermometers (object, machine, gauge); $\mathrm{u}_{\mathrm{tempw}}, \mathrm{u}_{\mathrm{tempN}}$ - standard uncertainty of temperature measurement (object, machine).

Other uncertainties were determined as follows:

$\mathrm{u}(\mathrm{I})$ - standard deviation of measurements; $\mathrm{u}\left(\mathrm{P}_{\mathrm{IP}}\right), \mathrm{u}\left(\mathrm{P}_{\mathrm{IS}}\right)=\frac{E_{g}}{\sqrt{3}}$, where $\mathrm{E}_{\mathrm{g}}-$ maximal permissible error of the arm (volumetric accuracy from catalogue) and laser scanner (accuracy from catalogue) respectively, under the assumption that maximum permissible errors have uniform distribution.

Uncertainties for each feature are presented in Table 6.

Table 6. Uncertainties for features.

\begin{tabular}{|c|c|c|c|c|}
\hline & \multicolumn{2}{|c|}{$\begin{array}{c}\text { Comparison of point measurements } \\
\text { and pseudo-scanning }\end{array}$} & \multicolumn{2}{c|}{$\begin{array}{c}\text { Comparison of point } \\
\text { measurements and non-contact } \\
\text { scanning }\end{array}$} \\
\hline & $\begin{array}{c}\text { point } \\
\text { measurements, } \\
\mathrm{mm}\end{array}$ & $\begin{array}{c}\text { pseudo-scanning, } \\
\mathrm{mm}\end{array}$ & $\begin{array}{c}\text { non-contact } \\
\text { measurements, } \\
\mathrm{mm}\end{array}$ & $\begin{array}{c}\text { point } \\
\text { measurements, } \\
\mathrm{mm}\end{array}$ \\
\hline cylinder $\phi 70$ & 0.042 & 0.039 & - & - \\
\hline cylinder $\phi 13.002$ & 0.025 & 0.024 & 0.105 & 0.026 \\
\hline cone $26.565^{\circ}$ & 0.029 & 0.026 & - & - \\
\hline distance 110.000 & - & - & 0.106 & 0.038 \\
\hline sphere $\phi 24.000$ & 0.031 & 0.026 & 0.083 & 0.033 \\
\hline
\end{tabular}

Values from Table 6 show that comparing point measurements and pseudo-scanning, similar results are obtained. However, uncertainties for point measurements are slightly higher. There is a different situation when point measurements and non-contact scanning are compared. Uncertainties for laser scanning are several times higher than for point measurements. There is a significant influence of factors like properties of surface or environmental conditions on results. The cube was covered with white powder to improve its scattering. This method is used by opticians. The thickness of coating was $0.010 \mathrm{~mm}$ which is one order of magnitude lower than the uncertainty of results.

\section{Conclusions}

The main purpose of the study was to carry out measurements using Coordinate Measuring Arm and analysis to check the significance of differences between the results. The first part included a comparison of point measurements of contact and pseudo-scanning measurements. In the second part, point measurements of contact and contactless scanning measurements were compared.

The first part showed that in results of statistical tests for some geometric features there are statistically significant differences between the studied methods of measurement at the 95\% confidence level. Results of statistical parameters (range and standard deviations) showed higher repeatability in the pseudo-scanning measurement for each measured geometric feature.

Differences between calculated values of standard deviations ranged from 0.003 to $0.010 \mathrm{~mm}$, while differences between calculated values of range from 0.013 to $0.035 \mathrm{~mm}$. In order to obtain higher accuracy during point measurement successive points gathering without 
breaking contact between the measuring probe and the measured element is recommended. Time required to perform point measurements and pseudo-scanning is similar.

For this reason it is recommended to choose the method with higher repeatability i.e. pseudo-scanning.

In the second part, preliminary experimental studies have already shown that the accuracy of the scanning measurements with a laser head is characterized by higher uncertainty of measurement compared to the method of contact. This was confirmed by results of this study, because the errors expressed by mean square deviation were several times higher and so for contactless measurement of the cylinder are $0.100 \mathrm{~mm}$, whereas for contact $0.012 \mathrm{~mm}$; similarly for the sphere these values were $0.077 \mathrm{~mm}$ and $0.023 \mathrm{~mm}$ respectively, and to determine the distance $0.099 \mathrm{~mm}$ and $0.022 \mathrm{~mm}$. Even greater differences exist in values of range - according to data from Table 3 and 4 the value of range for determining the sphere is 3.8 times higher for scanning measurements, and up to eight times for cylinder. Problems with geometrically complicated features were also confirmed by Sładek et al. [16, 17]. Authors at first explained this by the character of the measured object, which made of a nearly transparent material could not be proper for non-contact measurements and was covered by white powder [18]. However, further studies with a metal element gave similar results. The results differ significantly from the data from the catalogue, for which uncertainty on the level of $2 \mathrm{~s}$ as permissible errors for the scanner is $35 \mu \mathrm{m}$ and for the Arm $40 \mu \mathrm{m}$.

Moreover, based on analysis of data it was noted that from among all researched features the least standard deviation is appearing for the cylinder, after which it can be concluded that the Measuring Arm measures most accurately in the case when the rotation is appearing in the smallest number of joints, and the tactile probe moves insignificantly.

Statistical analysis confirmed with the probability of $95 \%$ that there are statistical significant differences between results of contact and contactless measurements.

The obtained results give a basis for further research in order to explain such big errors of scanning measurements. It would be necessary to carry out research having as a goal to determine the sources of error of scanning measurements. Analysis of sources of errors and their influence on the measurement would enable the compensation for environmental influences on the measurement.

One could also do research on how data processing influences the measurement result. Whether raw results significantly differ from those processed in Focus software.

The main direction of further research is to combine the two methods of measurement in such a way to maintain the advantages of both - accuracy of contact measurements and high density of points in non-contact measurements.

\section{References}

[1] Ratajczyk, E. (2005). Coordinate measuring technique. Publisher of Warsaw University of Technology, Warsaw. (in Polish)

[2] Ratajczyk, E. (2009). Robots and measuring centers. Measurement Automation and Robotics, (3), 5-9. (in Polish)

[3] Ratajczyk, E. (2009). Metrology in production engineering. Mechanik, (7), 619-626. (in Polish)

[4] Ratajczyk, E. (2009). Measuring arms - accuracy tests. Mechanik, 2, 104-107. (in Polish)

[5] Jakubiec, W. (2010). Estimation of uncertainty of coordinate measurements according to the B method. Key Engineering Materials, 437, 253-257.

[6] Jakubiec, W., Płowucha, W., Starczak, M. (2011). Analytical estimation of coordinate measurement uncertainty. Measurement. http://dx.doi.org/10.1016/j.measurement.2011.09.027

[7] www.nikonmetrology.com, www.smart-solutions.pl 
[8] ASME B89.4.22-2004 Methods for Performance Evaluation of Articulated Arm Coordinate Measuring Machines.

[9] Ratajczyk, E., Zawacki, M. (2008). Accuracy tests of measuring arms - is it possible to compare ASME and ISO standard requirements. In Proc. 8th International Conference - Coordinate measuring technique, Bielsko-Biała, Poland, 137-146.

[10] Poniatowska, M., Werner, A. (2010). Fitting spatial models of geometric deviations of free-form surfaces determined in coordinate measurements. Metrol. Meas. Syst., 17(4), 599-610.

[11] Bubnowicz, S., Łukianowicz, Cz. (2010). Assessment of measurement uncertainty of some geometrical quantities in coordinate measurements. Measurement Automation and Monitoring, (1), 2010, 4-5.

[12] Poniatowska, M. (2009). Research on spatial interrelations of geometric deviations determined in coordinate measurements of free-form surfaces. Metrol. Meas. Syst., 16(3), 501-510.

[13] Poniatowska, M., Werner, A. (2010). Simulation tests of the method for determining a CAD model of free-form surface deterministic deviations. Metrol. Meas. Syst., 17(4), 599-610.

[14] Dobosz, M. (2001). Computer-aided statistical analysis of test results. Academic Publishing House EXIT, Warsaw. (in Polish)

[15] Sładek, J. (2011). Accuracy of coordinate measurements. Publisher of Cracow University of Technology, Cracow. (in Polish)

[16] Sładek, J., Olszewska, M., Gąska, A., Ostrowska, K., Ryniewicz, A. (2012). Method of assessing the measurement accuracy performed by articulated arms equipped with optical scanning heads. Mechanik, 2, 133-135. (in Polish)

[17] Sładek, J., Sitnik, R., Kupiec, M., Błaszczyk, P. (2010). The hybrid coordinate measurement system as a response to industrial requirements. Metrol. Meas. Syst., 18(1), 109-118.

[18] Juras, B., Szewczyk, D. (2011). Accuracy of measurements performed on laser scanner. Advances in Science and Technology, (7), 29-36. (in Polish) 